\title{
A Statistical Model of Tropical Cyclone Tracks in the Western North Pacific with ENSO-Dependent Cyclogenesis
}

\author{
EMMI YONEKURA \\ Department of Earth and Environmental Science, Columbia University, New York, New York \\ TIMOTHY M. HALL \\ NASA Goddard Institute for Space Studies, New York, New York
}

(Manuscript received 9 August 2010, in final form 29 November 2010)

\begin{abstract}
A new statistical model for western North Pacific Ocean tropical cyclone genesis and tracks is developed and applied to estimate regionally resolved tropical cyclone landfall rates along the coasts of the Asian mainland, Japan, and the Philippines. The model is constructed on International Best Track Archive for Climate Stewardship (IBTrACS) 1945-2007 historical data for the western North Pacific. The model is evaluated in several ways, including comparing the stochastic spread in simulated landfall rates with historic landfall rates. Although certain biases have been detected, overall the model performs well on the diagnostic tests, for example, reproducing well the geographic distribution of landfall rates. Western North Pacific cyclogenesis is influenced by El Niño-Southern Oscillation (ENSO). This dependence is incorporated in the model's genesis component to project the ENSO-genesis dependence onto landfall rates. There is a pronounced shift southeastward in cyclogenesis and a small but significant reduction in basinwide annual counts with increasing ENSO index value. On almost all regions of coast, landfall rates are significantly higher in a negative ENSO state (La Niña).
\end{abstract}

\section{Introduction}

Typhoons in the western North Pacific Ocean (WNP) have potential to cause great damage on populated coastal areas in China, Taiwan, Japan, and the Philippines. The Pacific's high sea surface temperatures provide a fertile environment for tropical cyclogenesis. The Pacific consequently has the highest annual storm count of any ocean basin, averaging about 35 storms per year [from the International Best Track Archive for Climate Stewardship (IBTrACS) 1945-2007 averaged annual rates]. The landfall rate of tropical cyclones (TC) is one convenient diagnostic of the impact of TCs on coastal regions. Assessing the risk of landfall and assessing its variation with climate are important for coastal land use policy, disaster management, and financial decisions for insurers. Here, we describe a new statistical model for

Corresponding author address: Emmi Yonekura, Columbia University Department of Earth and Environmental Science, 2880 Broadway, New York, NY 10025.

E-mail: eyonekura@giss.nasa.gov
WNP typhoon genesis and tracks and apply the model to estimate landfall rates as a function of the state of El Niño-Southern Oscillation (ENSO).

Our approach is to develop and apply a basinwide statistical track model that simulates the full life cycle of a TC, following and expanding on the model of Hall and Jewson (2007) for the North Atlantic Ocean. The model is constructed on a basinwide dataset that is at least two orders of magnitude larger than the landfall data alone. A dataset of synthetic TCs that is many times as large as the historical set is generated, and the synthetic TC tracks are used to determine landfall rates. The major components of the model are genesis, track propagation, and lysis (death). The primary application is the effect of ENSO on cyclogenesis and how this effect influences landfall rates.

Thus, a key feature of the model is the sensitivity of the genesis component to ENSO state. Previous studies that have explored the relationship in the WNP between ENSO and cyclogenesis (Chan 1985, 2000; Saunders et al. 2000; Chia and Ropelewski 2002; Wang and Chan 2002; Camargo and Sobel 2005; Camargo et al. 2007a,b; Dong 1988) have found that during an El Niño year 
more TCs form in the southeastern part of the basin whereas during a La Niña year more TCs form in the northwestern part of the basin, concentrating nearer to the continent. Early studies indicate that this change is due to the change in the Walker circulation (Dong 1988). Camargo et al. (2007a) more recently used a genesis potential index to find that El Niño influences on relative humidity and low-level vorticity are responsible for the shift.

The shift in genesis location has implications on the rest of the TC life cycle. Tracks in the WNP tend to recurve farther north (above $35^{\circ} \mathrm{N}$ ) during El Niño years, resulting in more landfalls on Japan and the Korean peninsula. La Niña years tend to have more straight-moving tracks that make landfall on southern China, the Philippines, and Vietnam (Camargo et al. 2007b; Wang and Chan 2002; Elsner and Liu 2003; Wu et al. 2004; Fudeyasu et al. 2006; Saunders et al. 2000). Further, TC lifetime and intensity are also affected by ENSO state in the WNP. Studies (Camargo et al. 2007a; Camargo and Sobel 2005; Wang and Chan 2002; Chan and Liu 2004; Chan 2005, 2006) agree that El Niño years produce TCs that are more intense and last longer. The genesis location is partly accountable for this change because TCs that form in the southeastern part of the WNP have more time to intensify before making landfall and dissipating. Here, local Poisson regression is applied to model the shift in the geographic distribution of genesis with ENSO. Basinwide Poisson regression on ENSO state is used to model the basinwide TC frequency. The track model then allows these ENSOgenesis features to be projected onto eastern Asian landfall.

We begin by describing the data employed and follow with descriptions of the statistical method for each model component. Then the results are shown for track simulation and evaluation of the model using several diagnostics. The changes in regional landfall rates with ENSO state are then examined.

\section{Data}

The model is built on data from IBTrACS (Knapp et al. 2010). All of the WNP data from 1945 to 2007 are used to incorporate data from the beginning of routine aircraft reconnaissance. The data are in the form of 6-hourly storm-center position coordinates and maximum sustained wind speeds. IBTrACS was constructed as an effort to compile the data from multiple observational records from institutions across the globe. It is especially useful for the WNP where there are many databases with overlapping and sometimes conflicting records. The advantage of using these "best tracks" is that

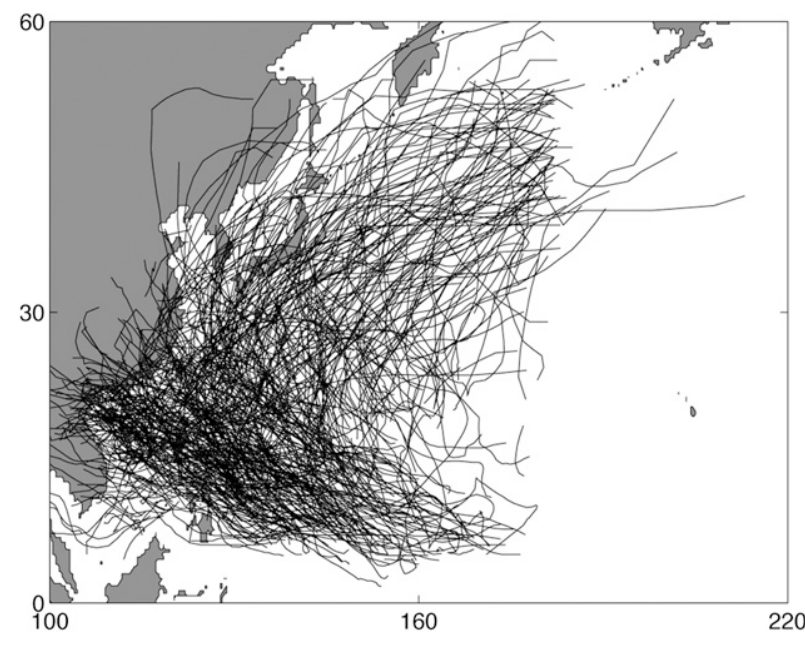

FIG. 1. Randomly selected $20 \%$ of the IBTrACS 2295 historical TC tracks from 1945 to 2007.

they consider the records from all institutions and reconcile storm omission and repetition.

The WNP has a high annual TC occurrence rate, totaling 2295 storms on which this analysis is based. These TC tracks are shown in Fig. 1. There is a clear overall pattern of storm tracks, with westward motion at low latitudes, recurvature, and eastward motion at mid- and high latitudes (Harr and Elsberry 1991). It is also clear that the historical tracks display large deviations from this mean path, which can be mimicked well stochastically.

For the genesis model, the Niño-3.4 index averaged over July-October (JASO) is used to indicate the ENSO state. These data are derived from the monthly sea surface temperature anomaly in the $5^{\circ} \mathrm{S}-5^{\circ} \mathrm{N}, 170^{\circ}-120^{\circ} \mathrm{W}$ region (Barnston et al. 1997) and are obtained from the National Centers for Environmental Prediction (NCEP) Climate Prediction Center.

\section{Methods}

The goal is to estimate TC landfall rates on WNP Asian coastlines and their sensitivity to ENSO state. Our approach is to construct a WNP-wide genesis-totermination statistical model of TCs. Other approaches to landfall risk assessment are possible, for example, models that are based solely on landfall data (Chan and Shi 2000; Elsner et al. 2006). One advantage to a basinwide model is the use of the much larger full-basin dataset. Landfall rates depend on the statistical properties of TCs, and these can be estimated by using historical TC data over the entire basin, in addition to the actual TC segments making landfall. Another advantage to a basinwide model is the increased physicality: the influence 

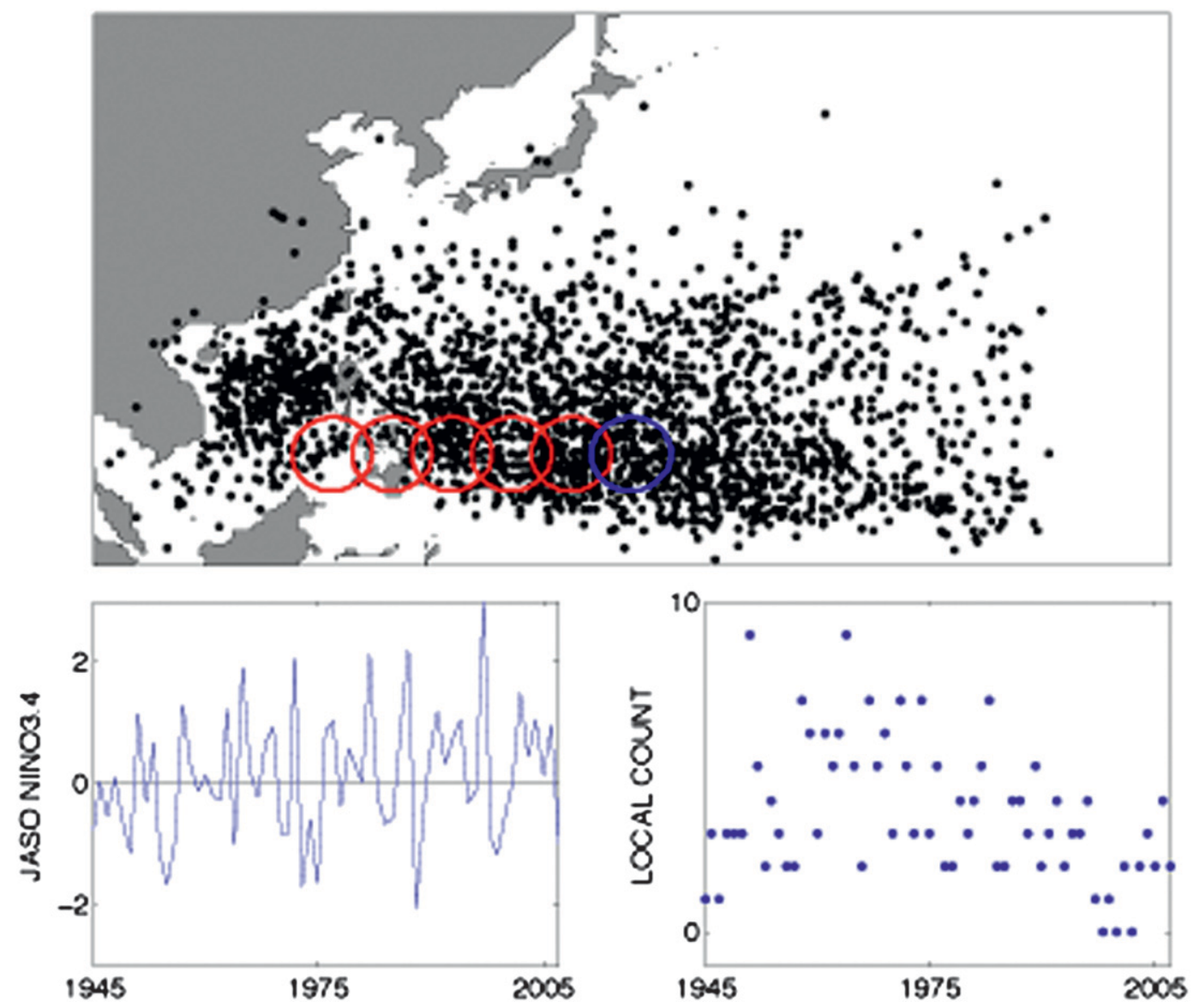

FIG. 2. Illustration of the local Poisson regression procedure for the genesis model. (top) Map of the historical genesis sites with examples of overlapping 370-km circles. Time series of the (bottom left) JASO Niño-3.4 index and (bottom right) annual genesis counts in the blue circle shown in the top panel.

of climate state on different components of TCs (e.g., genesis location, track propagation, and intensification) can be determined independently. A disadvantage is the increased complexity of the model, and the consequent increased possibility for model bias. Hall and Jewson (2007) explored quantitatively for a North Atlantic track model the trade-offs between increased precision (use of more data) and the potential loss of accuracy (increased chances for bias) relative to a model that uses solely landfall data.

Previous statistical track models include Drayton (2000) from the private sector and Darling (1991), Chu and Wang (1998), Vickery et al. (2000), Emanuel et al. (2006), James and Mason (2005), Rumpf et al. (2007), and Hall and Jewson (2007) from academic research. Not all employ a full statistical track model, and most focus on hurricanes in the North Atlantic. The basic approach to the model construction is local regression, closely following the work of Hall and Jewson (2007) for the North Atlantic. Local regression acknowledges that data geographically close to a location in question are most appropriate for estimating TC properties at the location. The length scale defining "close" is determined objectively in a manner to maximize the predictive skill of the model. Many features of our model are identical to those of Hall and Jewson (2007). These components are reviewed briefly in the following sections. Two components, genesis and the modeling of track errors, are distinct from those in Hall and Jewson (2007) and are described in more detail.

\section{a. Genesis}

The genesis model component determines how many TCs form in a year and where these TCs originate. The genesis model component from Hall and Jewson (2007) determined the number of storms forming annually in the North Atlantic by sampling a Poisson distribution whose mean is the historical average formation rate over the basin in the time period they analyzed. Where the 

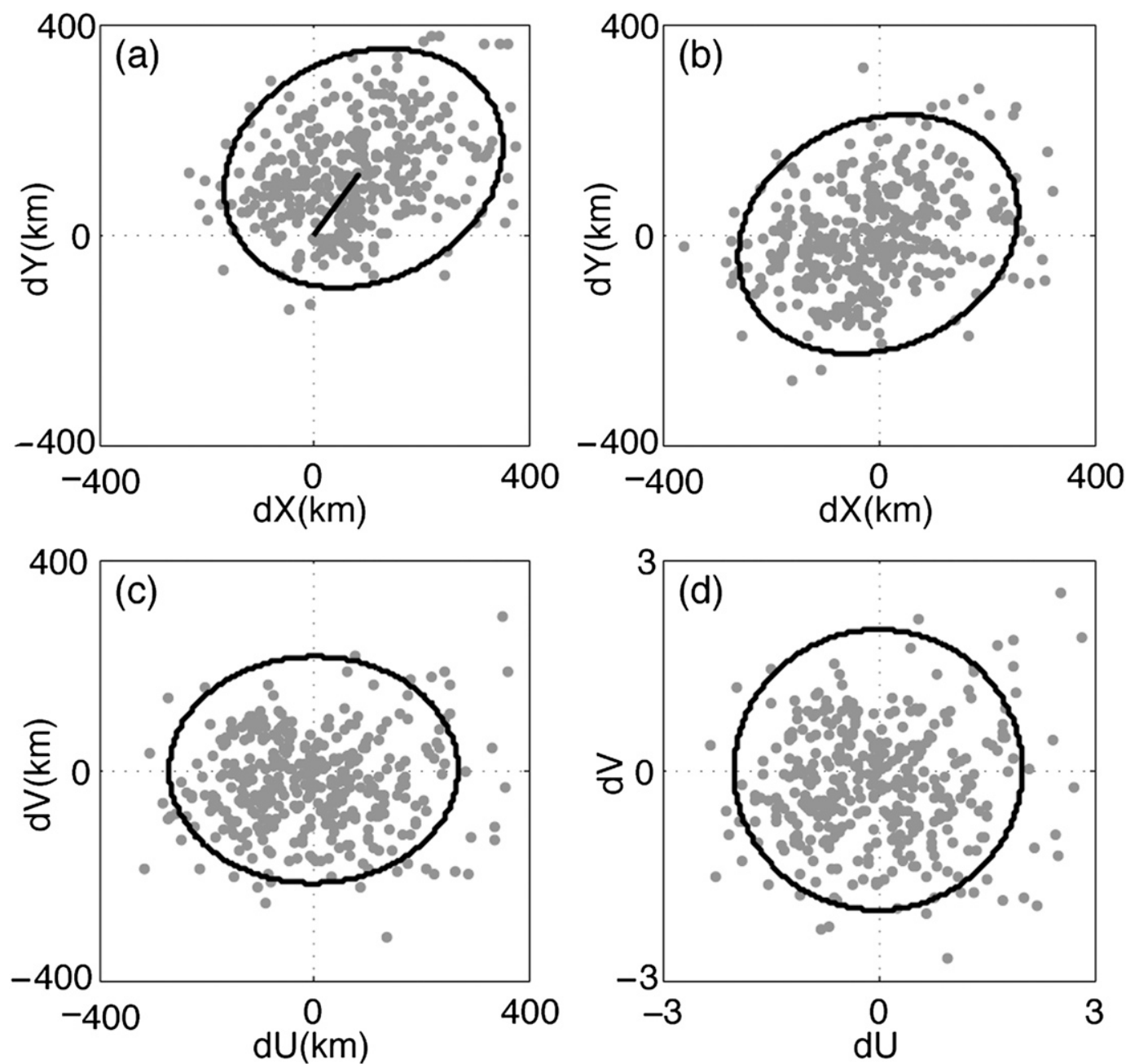

FIG. 3. (a) The $x$ and $y$ displacement values near grid point $34^{\circ} \mathrm{N}, 133^{\circ} \mathrm{E}$ are plotted against each other. The mean track increment vector is in black as well as the error ellipse defined by the principal axes. (b) As in (a), but now with the mean $x$ and $y$ increments removed. (c) As in (b), but now rotated to the covariance-ellipse principal axes. (d) As in (c), but now with the residuals divided by the rms variances along the major $(u)$ and minor $(v)$ covariance-ellipse axes. In (d) the deviations $u$ and $v$ are now uncorrelated and make up the standardized anomalies to be modeled independently.

TCs originated was determined by random sampling of an empirical kernel-density function. The Hall and Jewson (2007) genesis has no climate state sensitivity.

Because ENSO has been shown to influence TC formation in the WNP, it is included in this model. Genesis is still modeled as a Poisson process, but now the mean rate is dependent on ENSO state. Poisson regression is employed, which is the appropriate form of regression for count data (Sabbatelli and Mann 2007). The dependent variable is the annual formation rate (the mean Poisson rate) over the full domain, and it is related to the independent variable, the ENSO index, using a logarithmic link function: $\lambda_{j}=\exp \left(\beta_{0}+\beta_{1} \times \mathrm{ENSO}_{j}\right)$, where the $\beta$ are regression parameters and $\mathrm{ENSO}_{j}$ is the value of the ENSO state index for a specific year $j\left(\beta_{1}\right.$ is analogous to the slope, and $\beta_{0}$ is analogous to the $y$ intercept of linear regression). The regression parameters are chosen numerically to maximize the likelihood of the observed annual count time series, given the historical ENSO state index time series. In any particular year, the Poisson distribution associated with $\lambda_{j}$ is sampled to obtain a simulated TC count for the year.

Next we need to model where in the basin the TCs form. Local Poisson regression is used to simulate the local influence of ENSO on genesis. At each point on a $1^{\circ}$ grid the time series of annual TC formation counts within $L=370 \mathrm{~km}$ (see below for an explanation of the length scales) of the gridbox center is Poisson regressed 

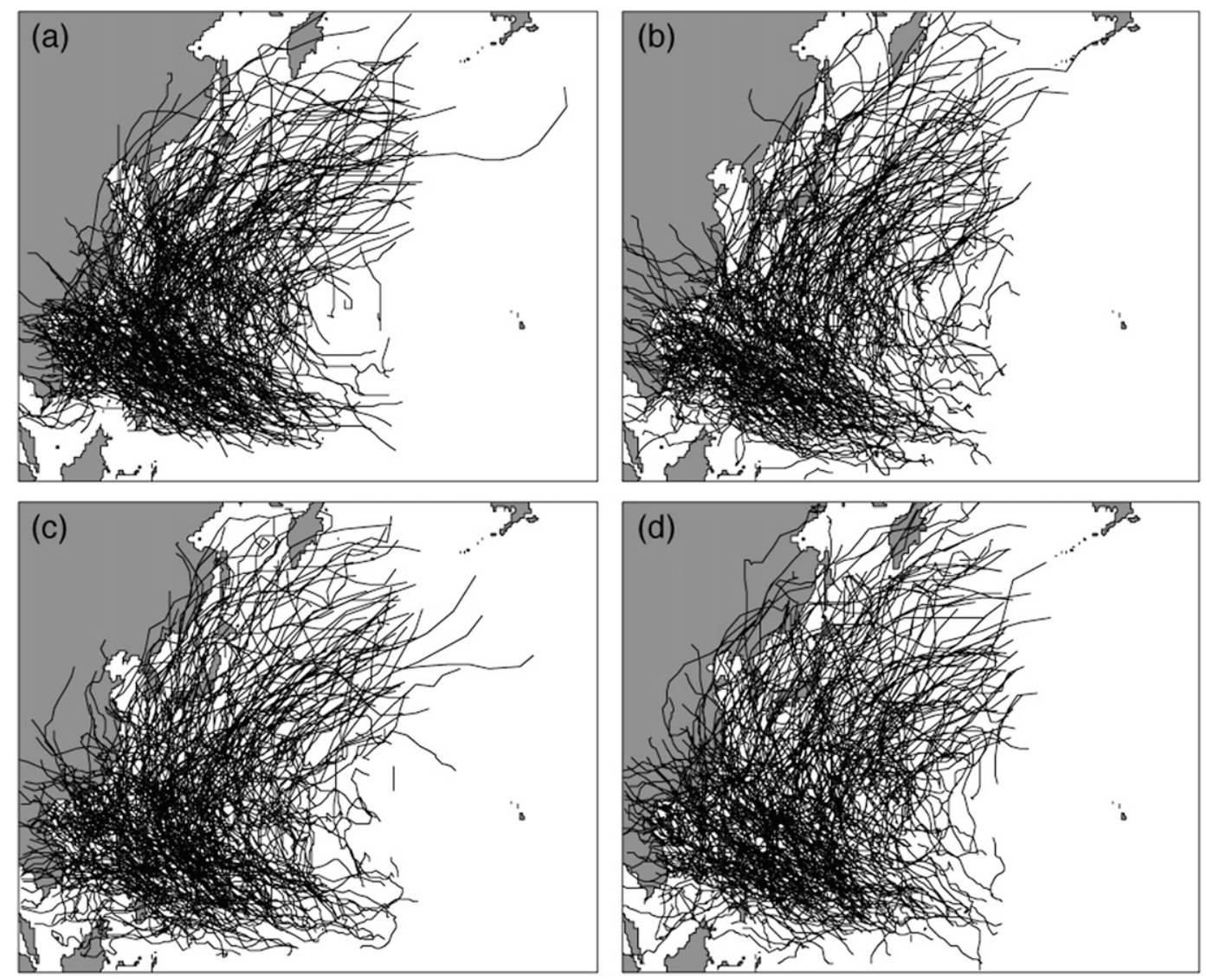

FIG. 4. (a) Historical tracks for 1945-2007 in the IBTrACS archive. (b)-(d) Three independent model realizations of the historical period. For all panels, a random $20 \%$ of the tracks were selected to better see the individual track shapes.

against the time series of ENSO state index. The rates are divided by the area of the $370-\mathrm{km}$ circle and multiplied by the gridbox area to obtain the mean rate on the grid box. In data-sparse regions regression is omitted, and the annual mean count is used for the Poisson rate. (If too few nonzero annual values are available, then the regression is unstable and is subject to large sampling error. The threshold value of 5 is somewhat arbitrary, striking a balance between avoiding local "hot spots" induced by sampling error and avoiding sensitivity to the threshold of the integrated basinwide rate.) Symbolically, then, in data-rich regions the $1^{\circ}$ Poisson rate is $\lambda_{j}^{\prime}(r)=A_{g} \exp \left[\beta_{0}^{\prime}(r)+\beta_{1}^{\prime}(r) \times \mathrm{ENSO}_{j}\right]$, where $r$ is the location, $A_{g}$ is the ratio of the area of the local $1^{\circ}$ gridbox area to the area of the local $370-\mathrm{km}$ circle, and the primes on $\lambda$ and $\beta$ indicate that the variables are now spatially variable.

Figure 2 illustrates the procedure. The IBTrACS genesis sites are shown on the map, along with several of the overlapping 370-km data circles. Inset are one circle's time series of ENSO values, the independent variable, and the annual TC formation count in the circle, the dependent variable. A probability density function (pdf) is then calculated by dividing the gridded local Poisson rates by the integrated sum of all local Poisson rates. Given $N$ total TCs from the basinwide genesis model, the pdf is sampled $N$ times to obtain the genesis location of storms in a simulated year with a given ENSO state.

\section{b. Track propagation}

TC propagation occurs by successive simulation of 6-h track displacements. The model for the 6-h increment is similar to that of Hall and Jewson (2007). The mean increment and the variance about the mean are computed locally by a weighted average of nearby historical increments, with the weighting length scale determined by out-of-sample minimization of forecast error (the mean) and maximization of likelihood (the variance). Also, as in Hall and Jewson (2007), the standardized errors are modeled as lag-1 autoregression [AR(1)] with 
(a)

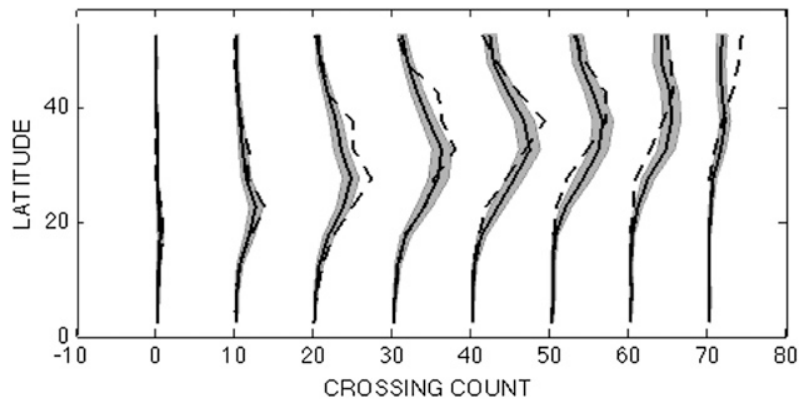

(b)

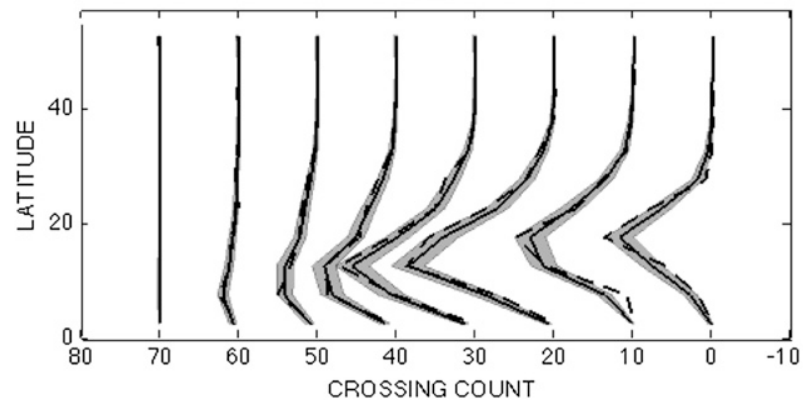

FIG. 5. Number of TCs crossing longitude lines $\left(110^{\circ} \mathrm{E}-180^{\circ}\right.$ equally spaced left to right) as a function of latitude. Unit increments on $x$ axis are crossings per $5^{\circ}$ latitude. (bottom) Westward crossings; (top) eastward crossings. Dashed lines are historical, and solid lines are ensemble mean simulation, with shaded gray area indicating \pm 1 std dev

autocorrelation coefficients computed by distanceweighted averaging of historical data.

The difference between our analysis and that of Hall and Jewson (2007) is the procedure to standardize the errors on which the $\operatorname{AR}(1)$ is applied. The $\operatorname{AR}(1)$ is applied independently to the vector components of the standardized track error, under the assumption that the components are uncorrelated. The Hall and Jewson (2007) standardized errors are in a frame of reference parallel and perpendicular to the local mean track increment, but we have found that this does not always result in independent error components. Here, the scatter about the mean increment is allowed to determine the principal axes of the local error ellipse, and we standardize the error in this local frame. Using the data-determined error ellipse frame better ensures that the components of the 2D standardized errors are uncorrelated.

The standardizing procedure is illustrated in Fig. 3. In the scatterplots of Figs. $3 a$ and $3 b$ (with and without means, respectively) it is clear that the $x$ and $y$ errors are correlated and, therefore, cannot be modeled independently. The errors are modeled with a generalized two-dimensional normal distribution, in which the variances in each dimension are allowed to be distinct and the motions in the two dimensions can covary (termed a "bivariate anisotropic correlated normal distribution"). The 2D scatter of such a distribution can be characterized by an error ellipse whose major axis makes an angle

$$
\alpha=\frac{1}{2} \tan ^{-1}\left(\frac{c_{x y} \sigma_{x} \sigma_{y}}{\sigma_{x}^{2}-\sigma_{y}^{2}}\right)
$$

with respect to the $x$ axis (line of constant latitude), where $\sigma_{x}$ and $\sigma_{y}$ are the rms error variances in the $x$ and $y$ (longitudinal and latitudinal) directions and $c_{x y}$ is the $x-y$ error correlation. Rotating the $x-y$ error into the principal-axes frame of reference (Fig. 3c) results in transformed errors, $u$ and $v$, that are independent and can be modeled as such. In Fig. $3 d$ the errors are divided by the rms variances in the principal axes to standardize them. Note that the degree of $x-y$ error correlation varies spatially and can be as high as 0.8 in some regions, illustrating the importance of accommodating it.

As in Hall and Jewson (2007) for the North Atlantic, here for the WNP we have checked for evidence of higherorder lags and for nonnormality in the errors. Higher lags are tested by performing multiple-variable regression of standardized errors against themselves at a number of lags: $i-1, i-2, i-3$, and so on. Only lag 1 was found to be significant. Normality was checked with quantile-quantile analysis. In both the $u$ and $v$ directions there are deviations from normality beyond 2 standard deviations. It was a concern that these "fat tails" might compromise the results, since normality is assumed in several stages of the analysis-most important, in the random forcing in the AR(1) model. To test sensitivity, simulations are performed in which archived historical errors are randomly sampled rather than using normal distribution forcing. If fat tails in the errors played a large role in track propagation, then one would expect a noticeable difference in simulation behavior between normal distribution forcing and archived-error sampling. In fact, there was no significant difference in track behavior according to the diagnostics described below.

\section{c. Lysis}

Lysis (the termination of tracks) is identical to that in Hall and Jewson (2007). The probability $P$ for track lysis (termination) in a 6-h time step at position $r$ is the ratio of the weighted sum of all terminal historical track points to the weighted sum of all track points. Lysis over ocean and lysis over land are separated using a $0.25^{\circ}$ mask to obtain different rates, because the physical processes on land and ocean are different. 

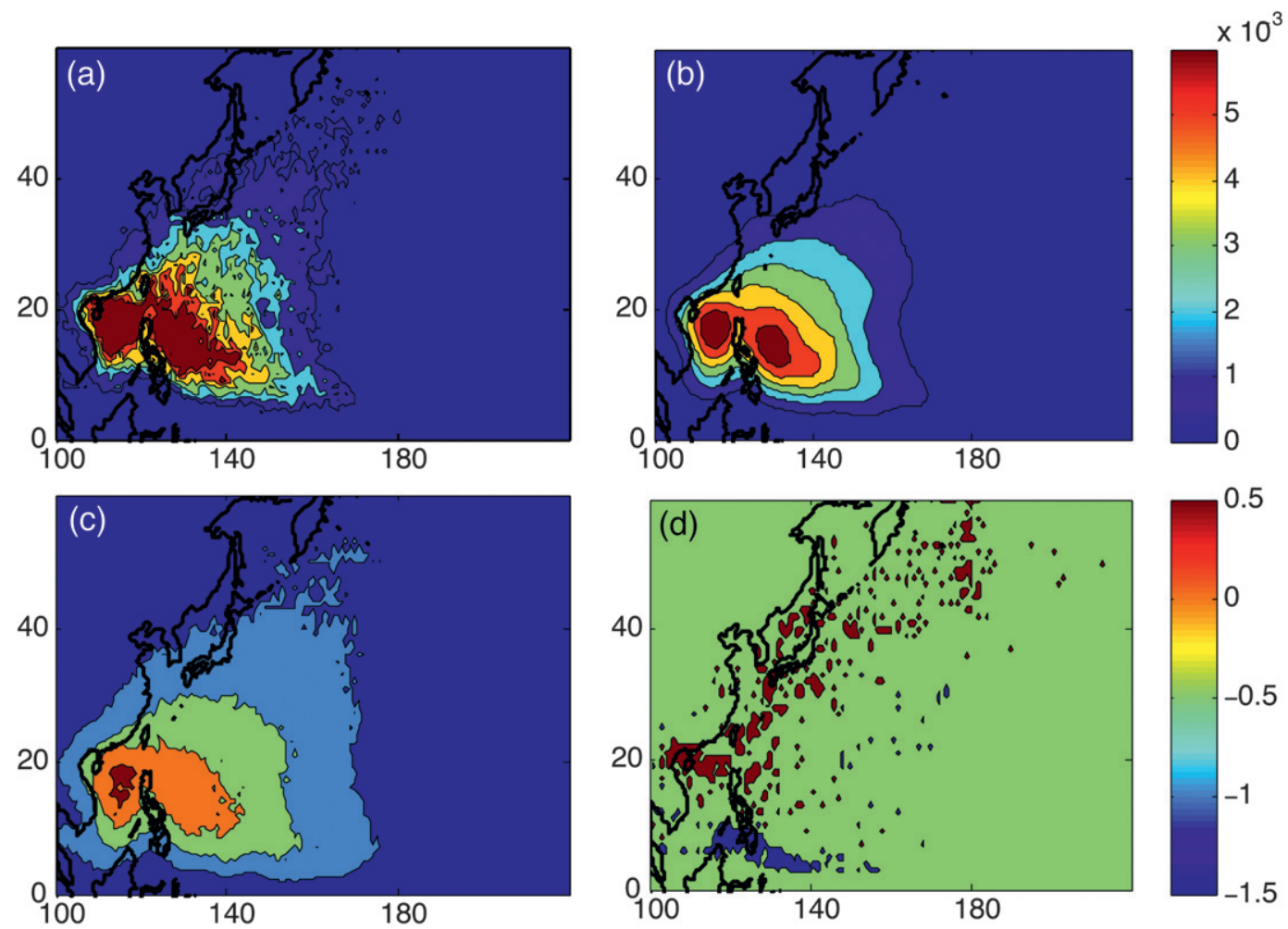

FIG. 6. (a) Historical track density (points per $1^{\circ}$ box in the 1945-2007 period), (b) 500-realization ensemble average track density, and (c) spread of the ensemble, shown by ordering track density values at each grid point and taking the difference between the $97.5 \%$ and $2.5 \%$ percentile values. (d) Red regions show where the historical track point density is above the $97.5 \%$ percentile level for that grid point. Blue shows where it is below the $2.5 \%$ percentile level. Green is where the historical values are bounded by the inner $95 \%$ of the ensemble.

\section{d. Optimized averaging scales}

In using historical data for each of the model components, the weight applied to a datum increases with geographic proximity to a simulation location in question (potential genesis site, current track location, or potential lysis site). The length scale of the weight determines the balance between the desire to use as many data as possible (a large scale) and the desire to resolve geographic structure as well as possible (a small scale). Hall and Jewson (2007) described year-by-year jackknife out-of-sample procedures to determine the length scales objectively, and we use their procedure here. The out-of-sample nature of the procedure (use of one data subset to build the model and use of the remainder subset to evaluate the model) ensures that the model is optimized for prediction.

Because we are working here in a different basin with different data volumes and different geographic distributions of statistical TC properties, the length scales obtained are not identical to those of Hall and Jewson (2007). We obtain $370 \mathrm{~km}$ for the local Poisson regression for genesis, $220 \mathrm{~km}$ for ocean lysis, $830 \mathrm{~km}$ for land lysis, $220 \mathrm{~km}$ for the mean track, and $260 \mathrm{~km}$ for the track variance. As illustrated in Hall and Jewson (2007), use of scales that are smaller than the optimal scale results in simulations whose features have unwarranted fine spatial structure. Use of scales that are larger than optimal results in excessive smoothing of true geographic structure. An optimal length scale for the track-error autocorrelations was not obtained. We used $500 \mathrm{~km}$ and found little sensitivity to varying this value.

\section{e. Simulation}

There are four steps to simulate the historical period (1945-2007):

1) For each of the 63 years, both the basinwide Poisson rate and a grid of local Poisson rates are calculated using the historical ENSO state of the respective year. The associated Poisson distribution for the basinwide Poisson rate is sampled to give an annual count $N$ for the basin. The local Poisson rates are used to calculate a normalized pdf that is sampled $N$ times to give the locations of genesis. 


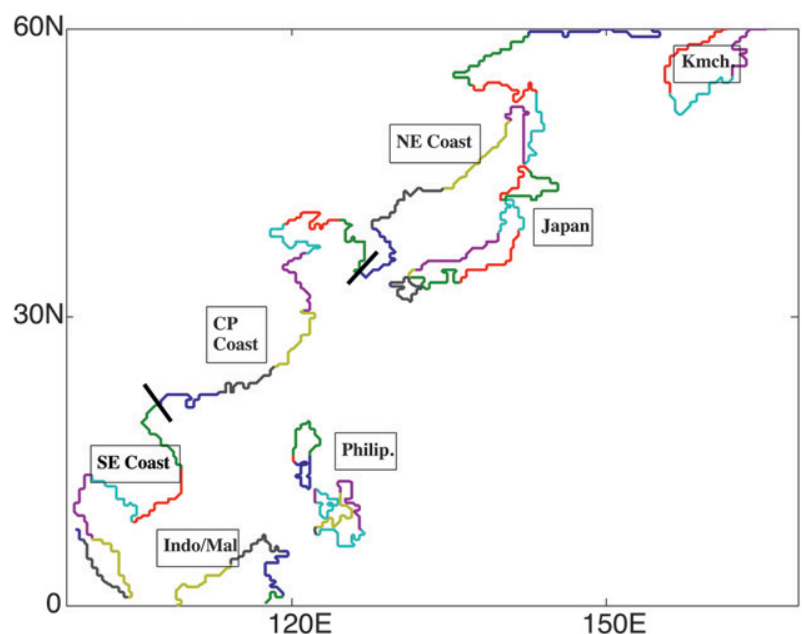

FIG. 7. Map of the 47 segments lining the main coast and significant other landmasses. Political regions are also marked for landfall analysis.

2) Tracks propagate from the genesis in 6-h time steps. At each position, the $\mathrm{AR}(1)$ model gives the standardized error $u_{i}=c_{u} u_{i-1}+s \varepsilon$, where $c_{u}$ is the autocorrelation coefficient, $\varepsilon$ is the standard normal random, and $s=\left(1-c_{u}^{2}\right)^{1 / 2}$ is the magnitude of the random term (Hall and Jewson 2007). A similar expression holds for the $v_{i}$, the perpendicular component of the error. (For the first step $c_{u}=c_{v}=0$.) The errors are then multiplied by the variances in the local principalcomponent reference frame and rotated back to the longitude-latitude frame, and the means are added, resulting in the next track increment. The simulated TC position is updated.

3) At each position, lysis is checked by comparing the local lysis probability $P$ with a uniform random draw $R$ from 0 to 1 . The track is terminated if $P>R$.

4) The procedure is repeated for all storms produced by the genesis model for each simulated year, and then for the desired number of simulations of the 19452007 period.

In the analysis that follows, 500 such simulations were used. This constitutes the ensemble of simulations of the 1945-2007 period. The model is stochastic, and therefore each simulation is distinct.

\section{Results}

\section{a. Model diagnostics}

Figure 4 shows the historical tracks and three realizations of the 63-yr simulations-for example, three members of the ensemble of simulations. In qualitative terms, the simulated tracks reproduce the large-scale

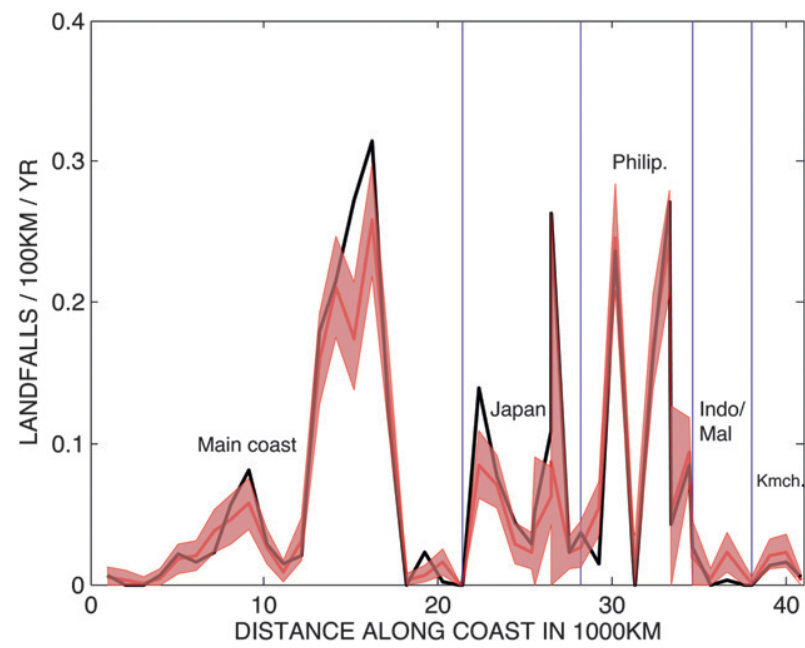

FIG. 8. Landfall rates along the eastern Asian coast. The figure shows the historical (black) and ensemble mean (red), with red shading for the inner $95 \%$ of the simulated landfalls. Units are counts per 100 kilometers per year.

features of the historical tracks, propagating westward in the subtropics and eastward in midlatitudes. We aim to evaluate the model more quantitatively. The model performs "well" on a diagnostic to the extent that the historical value of the diagnostic falls inside some confidence band of the range of simulated values of the diagnostic (e.g., the middle 95\%). That is, a historical value should appear to be a typical draw from the ensemble of simulated values, rather than an outlier. Where this is not the case, the model is biased with regard to the diagnostic. Here, the model is evaluated in this way with respect to three diagnostics: 1) track crossings of lines of constant longitude, 2) spatial maps of track density, and 3) landfall rate and its geographic distribution.

It is important to note that the model is not fit, or trained, to these diagnostics. The large-scale behavior of simulated tracks emerges from the point-by-point stochastic modeling of genesis and the step-by-step stochastic modeling of 6-hourly track increments. The model parameters (the averaging length scales) are chosen objectively to optimize the point and step processes.

Figure 5 shows eastward and westward track crossings over lines of constant longitude (counts in $5^{\circ}$ latitude bins) from the historical record and the simulations. Shown are means across the 500-member simulation ensemble and the inner $95 \%$ range about these means. This range about the model mean is used as the measure of significant difference between modeled and historical track crossings. Where the historical curve falls outside the spread about the model mean, the model is biased, 
NE Coast
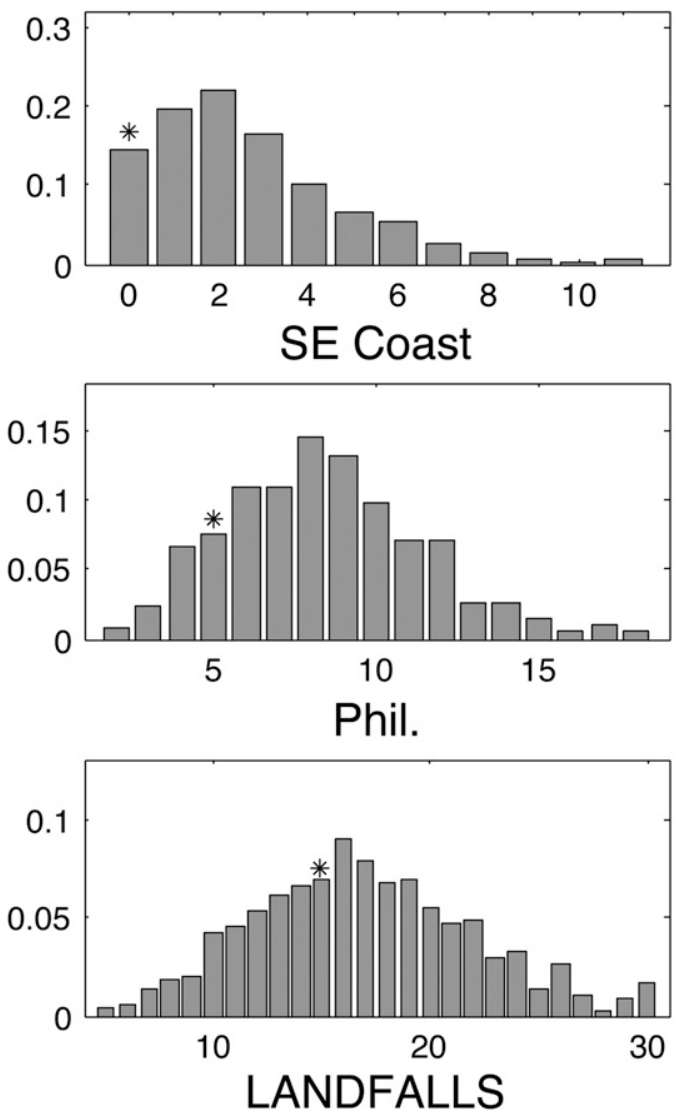

CP Coast
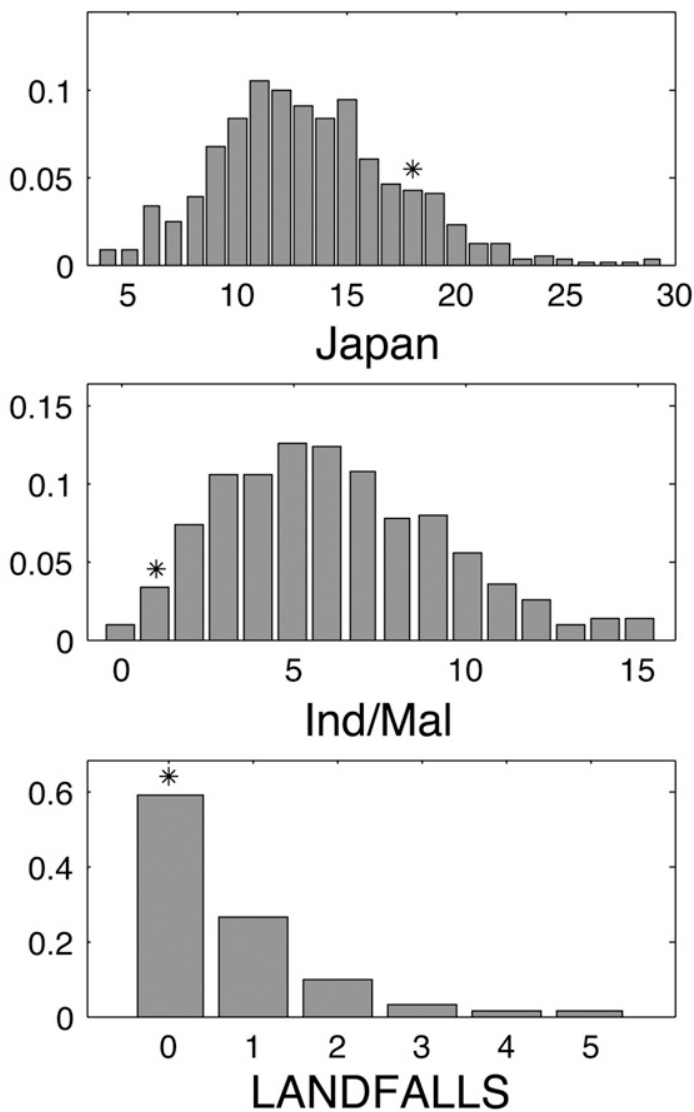

FIG. 9. Out-of-sample test using landfall data from the 2008-09 period. For each political region, the bars represent the distribution of landfalls resulting from 1000 model simulations of the out-of-sample period. The asterisk represents the historical (IBTrACS) values for landfalls in 2008 and 2009.

according to this confidence standard. The behavior of the mean track is to move westward between $5^{\circ}$ and $20^{\circ} \mathrm{N}$ and eastward between $20^{\circ}$ and $45^{\circ} \mathrm{N}$. As can be seen in the figure, the distribution of east and west track crossings closely follows that of the historical crossings, except for a few scattered regions where the historical curves lie outside the model spread.

Track density (number of 6-h TC points per unit area) is also used as a diagnostic. Figures $6 a$ and $6 \mathrm{~b}$ show the historical and ensemble mean track density, respectively. The overall density distribution is well reproduced by the model, but the model overestimates the maximum in density around the Philippines and underestimates it from Southeast Asia to Japan. Figure 6c shows the spread of the simulation ensemble-for example, the difference between the model's $2.5 \%$ and $97.5 \%$ percentile track density value at each grid point. This range has a maximum where the observed track density maximum appears. Figure $6 \mathrm{~d}$ shows a measure of the significance of the model-observation difference: regions where the observed track density is above the 97.5\% simulation percentile level are red, and regions where the observed track density is below the $2.5 \%$ level are blue. The regional model disagreements and the difference in magnitude of the maximum track density are significant by this standard. We are currently exploring the reasons for these biases. In other regions the model shows no coherent bias.

Landfall risk assessment is the ultimate application of the model, and therefore examining landfall rates of the simulations is a key diagnostic. The coastline is divided into segments that are approximately $1000 \mathrm{~km}$ long, covering the Asian mainland; Japan; the Philippines; Indonesia and Malaysia; and Kamchatka, Russia, as illustrated in Fig. 7. A landfall occurs on a segment if a track crosses the segment heading from ocean to land. The landfalls are accumulated and plotted as a function of distance along the coastlines in units of landfalls per year per $100 \mathrm{~km}$ of segmented coastline. Shown in Fig. 8 are the historical landfall rates, 
the model ensemble mean landfall rate, and the middle $95 \%$ range of simulated rates across the ensemble.

There is a high degree of structure in the geographical distribution of landfall rates, even within regions of high track density, much of which can be understood in terms of the relative orientation of coastline segment and the direction of the mean track. If a coastline segment is parallel to the local mean track then a landfall is relatively unlikely, although not impossible, given random motions about the mean. If a coastline segment is perpendicular to the local mean track direction, then a landfall is relatively likely. Overall, the model captures the geographical distribution: the local maxima and minima of the landfall rate are well matched. The model exhibits low landfall biases in certain regions, however, consistent with behavior seen in the track density. Historical landfall counts are above $97.5 \%$ of the model simulations on the coast of China as well as Japan; that is, the model displays a low landfall bias on these regions. The model performs well in matching the historical landfalls for the Philippines, and it performs well on other less-active regions.

Last, 1000 simulations of 2008-09, two out-of-sample years, are performed using the observed values of the JASO Niño-3.4 index. The landfall rates are computed for political regions defined in Fig. 7, and the distributions are compared with observed landfall rates for 2008-09 (Fig. 9). Results show that the observed rates occur within the modeled distribution.

\section{b. ENSO dependence}

We now turn to the role of ENSO in WNP TC genesis and, through genesis, its impact on landfall rates. The results for ENSO's influence on total genesis and its geographic distribution confirm those of previous studies (Chan 1985, 2000; Chia and Ropelewski 2002; Wang and Chan 2002; Camargo et al. 2007a). The coefficients from the local Poisson regression are shown in Fig. 10. Notice that the $\beta_{1}$ coefficient that multiplies the ENSO state variable changes sign from positive in the southeastern part of the basin to negative in the northwest. The implication on genesis rates is further elucidated in Figs. $11 \mathrm{a}-\mathrm{c}$, which show the normalized pdfs produced using the distribution of annual formation rates from the Poisson regression for three values of ENSO state index: $+2 \sigma, 0$, and $-2 \sigma$, where $\sigma$ is 1 standard deviation. There is a northwestward shift in formation and an increase in the region west of $140^{\circ} \mathrm{E}$ and south of $30^{\circ} \mathrm{N}$ from El Niño to La Niña states. The historical genesis sites at corresponding ENSO states are shown in Figs. $11 \mathrm{~d}-\mathrm{f}$, and the shifting behavior is evident. This shift is seen most clearly in Fig. 12, which is a map of the difference El Niño $(+2 \sigma)$ minus La Niña $(-2 \sigma)$.

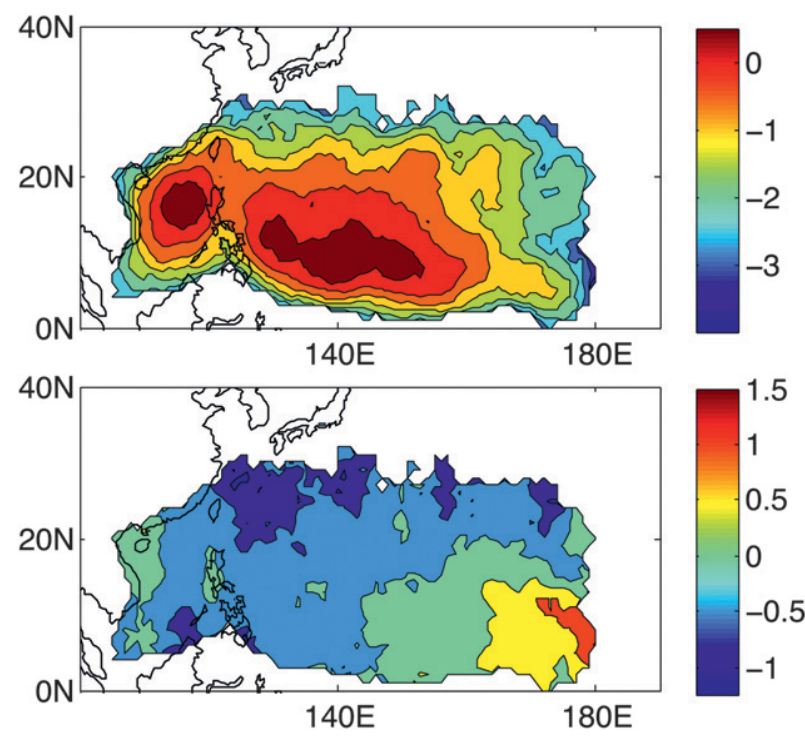

FIG. 10. Map of the local beta coefficients (top) $\beta_{0}$ and (bottom) $\beta_{1}$ calculated from local Poisson regression of genesis points and ENSO state.

The dependence of the basinwide annual count on ENSO (drawn from the Poisson distribution for the entire basin) is shown in Fig. 13a. The annual count decreases from 39 to 34 per year with a shift from $-2 \sigma$ to $+2 \sigma$ in ENSO. Given the indicated $95 \%$ range of the annual counts, this change is barely significant. Figures $13 \mathrm{~b}-\mathrm{d}$ show the annual counts as functions of ENSO state for subregions of the basin indicated in Fig. 12: the main development region $\left(\mathrm{MDR} ; 10^{\circ}-20^{\circ} \mathrm{N}, 110^{\circ}-\right.$ $\left.160^{\circ} \mathrm{E}\right)$, the "cool" region, and the "warm" region. In the MDR an ENSO change from $-2 \sigma$ to $+2 \sigma$ decreases the Poisson rate by five storms per year. In the cool region, the same $+4 \sigma$ ENSO change decreases the Poisson rate by roughly three storms per year. By contrast, the warm region has statistically more genesis activity during El Niño conditions: a $-2 \sigma$ to $+2 \sigma$ ENSO shift causes an increase of about three storms per year. Note that the Poisson regression model is not linear; genesis sensitivity to a specified change in ENSO depends on the absolute ENSO value about which the change occurs.

To determine the significance of these ENSO-genesis sensitivities, a jackknife test is performed. The counts are Poisson regressed on ENSO over the basin 10000 times, in each case randomly dropping $13(20 \%)$ of the data years used in the regression. In "delete- $d$ jackknife" the point is to choose a $d>1$ to increase the number of data subsets across which a statistic can be computed. For $d=1$ there would be 63 (1945-2007) subsets of 62 years each, which is not enough to examine the tails of the distribution-for example, to estimate 

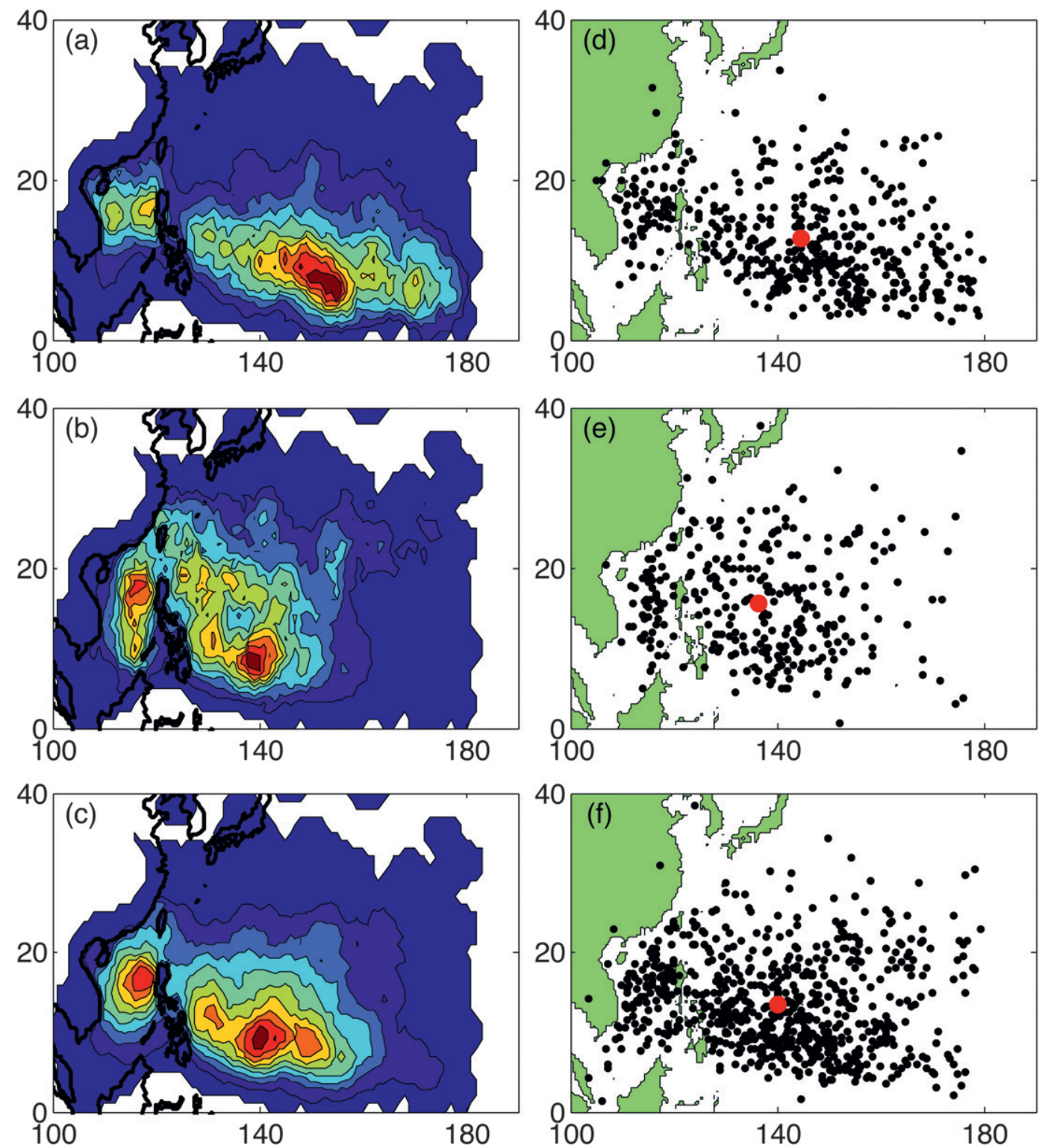

FIG. 11. (left) Maps of normalized probability distribution function from local Poisson rates, given the JASO Niño3.4 index values of (a) $2 \sigma$, (b) $-2 \sigma$, and (c) 0 . (right) The corresponding IBTrACS genesis sites for years with JASO Niño-3.4 index values of (d) greater than $\sigma$, (e) less than $-\sigma$, and (f) less than $\sigma$ but greater than $-\sigma$. The red point marks the mean genesis location.

the $2.5 \%$ and $97.5 \%$ confidence bounds. For $d=13$ there are $63 ! /[13 !(63-13) !] \approx 10^{12}$ subsets of 50 years each. On the other hand, $d$ should not be chosen to be so large that each subset is too small to allow one to perform regression. The value $d=13$ is a compromise.

In Fig. 12, El Niño-minus-La Niña differences are deemed significantly different than zero at a given location if the inner $95 \%$ of the differences across the jackknife set have the same sign. Locations where this is true are indicated in Fig. 12 with plus symbols. The regions of largest ENSO sensitivity are all significant by this measure. The same jackknife test is used to put confidence bounds (middle 95\%) on formation rates as a function of ENSO (Fig. 13). These bounds suggest that ENSO sensitivity is significant on a regional basis. The basinwide decline in formation rates with increasing ENSO state (Fig. 13a) is fractionally smaller than the regional changes because of cancellation due to opposing sensitivities in the warm and cool regions. To test the significance of basinwide sensitivity, the distribution of $\beta_{1}$ coefficients (the coefficient multiplying the ENSO value) is examined across the set of jackknife basinwide 


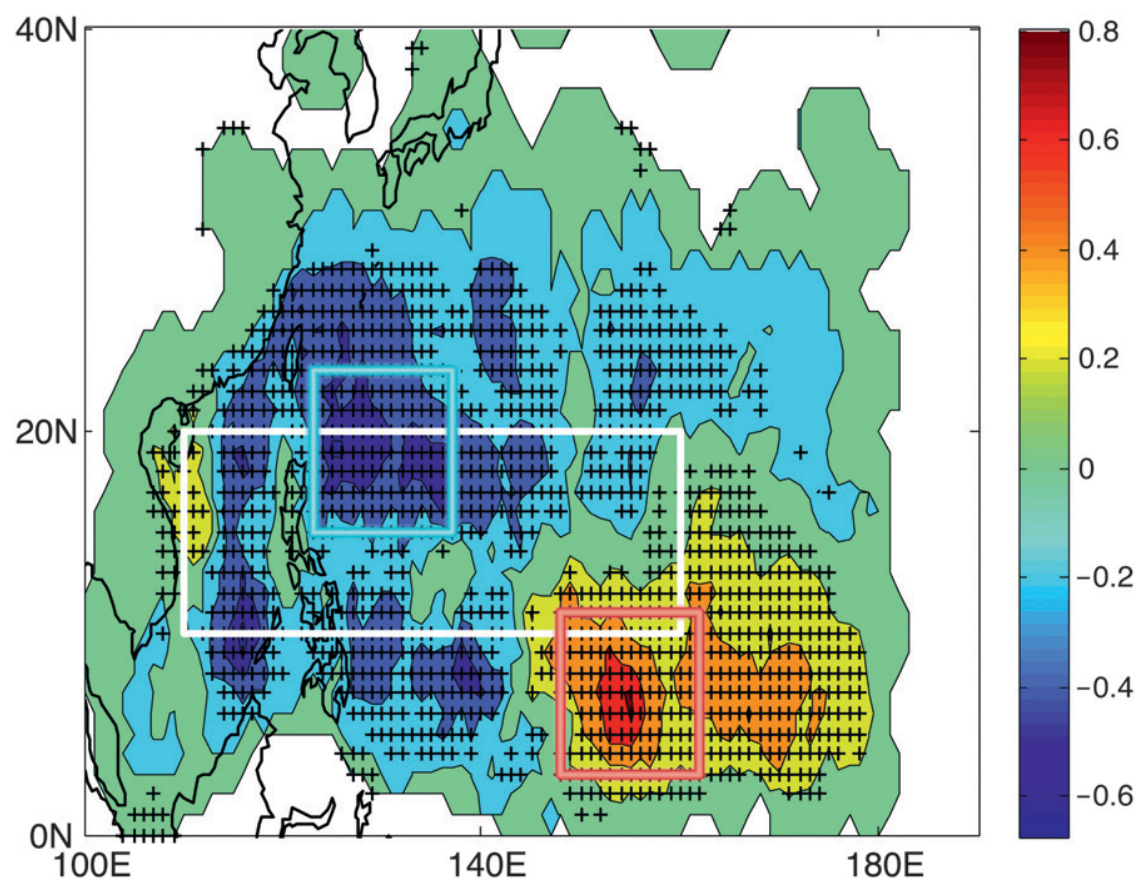

FIG. 12. Pdf difference of using ENSO state anomaly of 2 and -2 . The crosses mark the regions where the sign of the difference remains the same for the inner $95 \%$ of the bootstrap reconstructions of the genesis model that dropped out a random $20 \%$ of the data. The white box defines the MDR, the red box denotes the warm region, and the blue box denotes the cool region.

regressions. The $2.5 \%$ and $97.5 \%$ percentile values of these $\beta_{1}$ are roughly -0.06 and -0.01 , respectively, indicating that the basinwide formation decline with ENSO is significant. Thus, although we agree with the findings of Camargo and Sobel (2005) and Wang and Chan (2002) that basinwide formation sensitivity to ENSO is fractionally miniscule in comparison with regional sensitivity, we find the small sensitivity to be significant.

Both the change in basinwide rates and the shift in distribution with ENSO have consequences for landfall rates. During a La Niña event more TCs form in the basin and they form closer to the Asian coast. The impact of ENSO on landfall rates is shown in Fig. 14. Fifty model simulations of 1945-2007 are performed, each with a different fixed value of ENSO. The ensemble mean landfall profile is computed for each ENSO value. For most regions La Niña results in higher landfall than does El Niño. This is illustrated in Table 1, where the landfall rates of several political regions defined in Fig. 7 are shown for different ENSO states. For example, the central Pacific (CP) Asian coastline has a landfall rate increase of $40 \%$ for an ENSO change from $-2 \sigma$ to $+2 \sigma$. For the same change, the Philippines show a $9.5 \%$ landfall rate increase.
To highlight better the regional effects of ENSO state on landfall rates, Fig. 15 shows the fractional change in landfall rates for regions defined in Fig. 7. All regions show a decrease in fractional change with increasing value of ENSO state index. The change is most drastic from a strong La Niña event to a neutral state for Indonesia/Malaysia, the northeastern mainland coast, and Japan. This result does not agree with previous work (Wu et al. 2004; Saunders et al. 2000; Elsner and Liu 2003) that finds increased landfalls on Japan, the Korean peninsula, and northern China during El Niño events.

\section{Discussion}

We have developed a new statistical model for WNP typhoon tracks and genesis on the basis of IBTrACS data. The primary application is estimating Asian typhoon landfall rates on a regional scale and their sensitivity to ENSO. Model parameters (length scale in the local regression) are determined by an out-of-sample procedure to maximize the model's ability to forecast. The model captures well the overall features of WNP typhoon genesis and propagation, realistically reproducing both mean structure and pseudorandom variation 
(a)

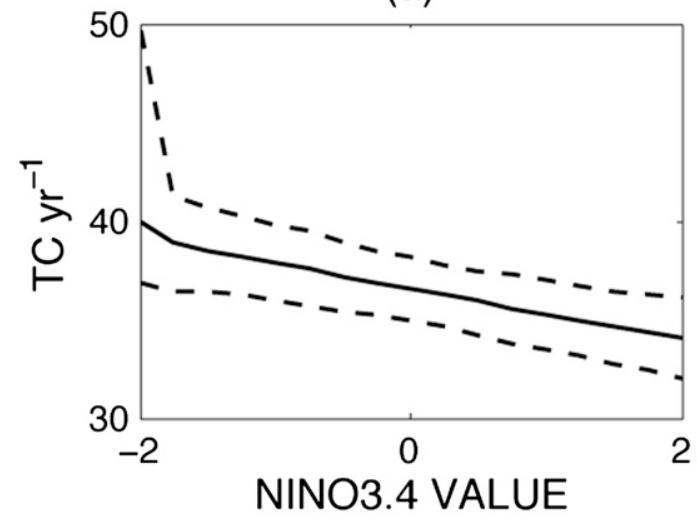

(c)

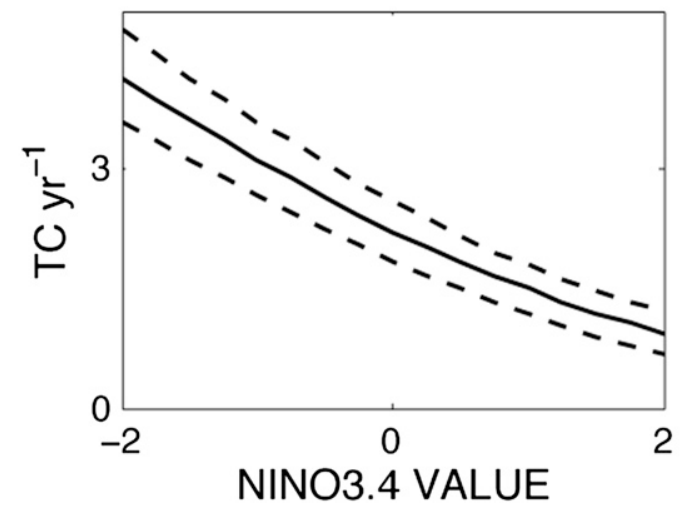

(b)

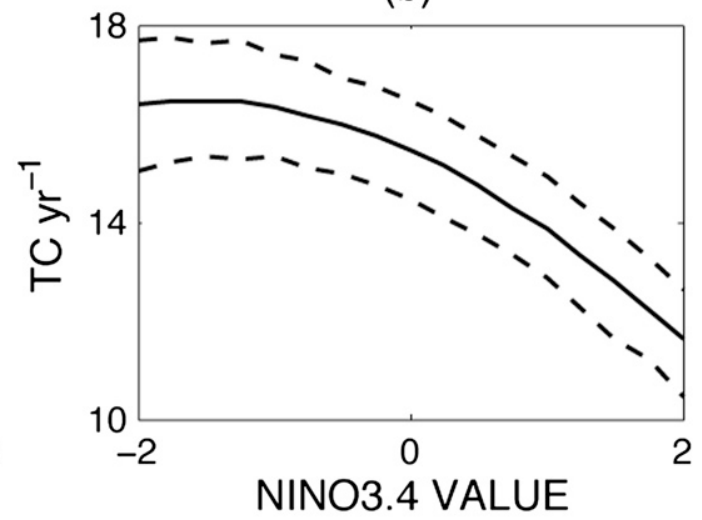

(d)

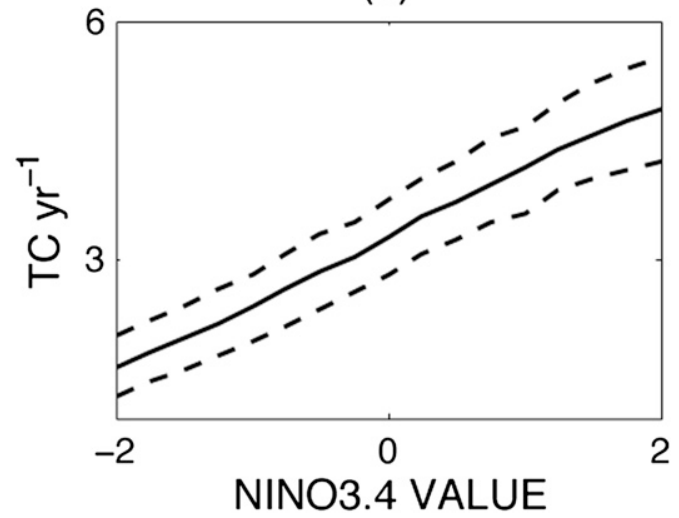

FIG. 13. Annual formation counts for different phases of ENSO for the (a) entire domain, (b) MDR, (c) cool region, and (d) warm region as defined in Fig. 12. Dashed lines indicate the inner $95 \%$ range from the jackknife method, and the solid line is the mean of 1000 jackknife versions of the genesis model.

about the mean. The geographic distribution of landfall rates is reproduced well in both historically active regions and in less-active regions. The realistic behavior in lessactive regions (e.g., Indonesia and Malaysia) is encouraging: a track model should be most advantageous over a model that is based solely on historical landfalls in regions where landfalls are rare.

The analysis reveals some model biases whose sources remain to be isolated and corrected. The discrepancy in track point density indicates that the model may be moving the storms too slowly to reach the coastal areas. This gives storms more chance to suffer lysis before making landfall, resulting in the low landfall biases seen for certain regions. The model also places roughly $5^{\circ}$ too far south the latitude of maximum eastward storm propagation in midlatitudes (Fig. 5a). Many of the historic storms in this region have undergone extratropical transition, which may affect their propagation but has not been explicitly considered here.

The genesis model has been constructed to be sensitive to ENSO. Regions of peak formation shift southeastward during El Niño and northwestward during La
Niña, in agreement with results of previous studies. The combined effects of a marginally significant increase in overall storms and formation closer to land result in more landfalls during La Niña. Important ENSO effects

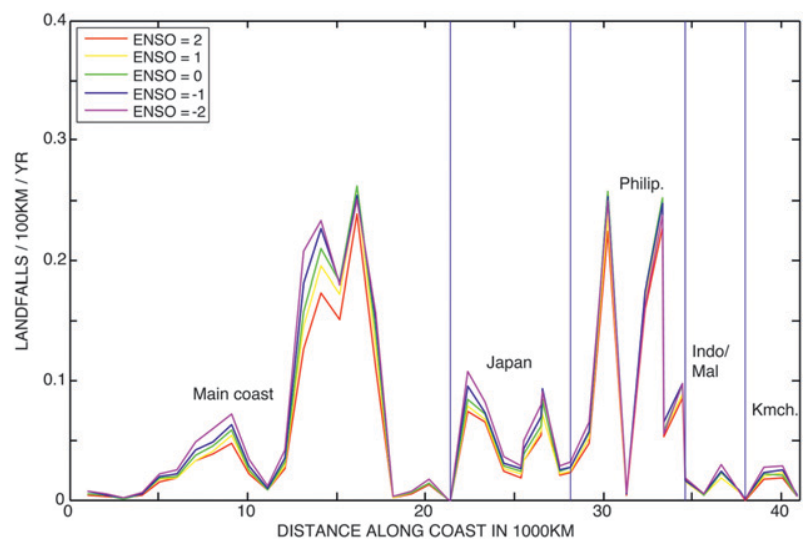

FIG. 14. Landfall rates from 50-realization model runs of the historical period given a stationary value of JASO Niño-3.4 anomaly. Positive anomalies are El Niño years, and negative anomalies are La Niña years. 
TABLE 1. Landfall rates, in units of landfalls per year per $100 \mathrm{~km}$ for specific political regions, given different Niño-3.4 JASO values. The bottom row shows the change from a +2 to a -2 phase of ENSO. The last column gives the genesis rates, in TC per year, for each ENSO state.

\begin{tabular}{cccccccc}
\hline \hline ENSO state & SE Asia & CP Asia & NE Asia & Japan & Philippines & All & Genesis rates \\
\hline 2 & 0.06 & 0.08 & 0.01 & 0.04 & 0.12 & 0.05 & 34.04 \\
0 & 0.07 & 0.10 & 0.02 & 0.05 & 0.14 & 0.06 & 36.53 \\
-2 & 0.07 & 0.11 & 0.02 & 0.06 & 0.13 & 0.07 & 39.21 \\
\% change* & 17.82 & 40.80 & 47.58 & 40.82 & 9.51 & 26.26 & 15.19 \\
\hline
\end{tabular}

* For rate $r, \%$ change is $[r(\mathrm{ENSO}=-2)-r(\mathrm{ENSO}=+2)] / r(\mathrm{ENSO}=+2)$.

remain to be included in the model, however, which may modify these results. Other studies (Wang and Chan 2002; Elsner and Liu 2003; Camargo et al. 2007b) argue that TC track shapes are sensitive to ENSO. Some of this effect is included in the sensitivity of the genesis distribution, because TCs forming in different regions will follow different trajectories according to the model. There may be additional influence that is currently not included that could be introduced by explicitly adding ENSO sensitivity to the track propagation component of the model. This may help to account for the differences in landfall sensitivity to ENSO found in this study when compared with previous studies. One crucial aspect is that an intensity model has not yet been implemented, and all landfalls are treated equally. TCs in El Niño years form farther from the coast and, therefore, have more time to intensify before making landfall (Wang and Chan 2002; Chan and Liu 2004; Chan 2005, 2006; Camargo and Sobel 2005; Camargo et al. 2007a). Thus, even though La Niña years have more overall landfalls, El Niño years are more likely to produce the intense landfalls that dominate risk-assessment considerations.

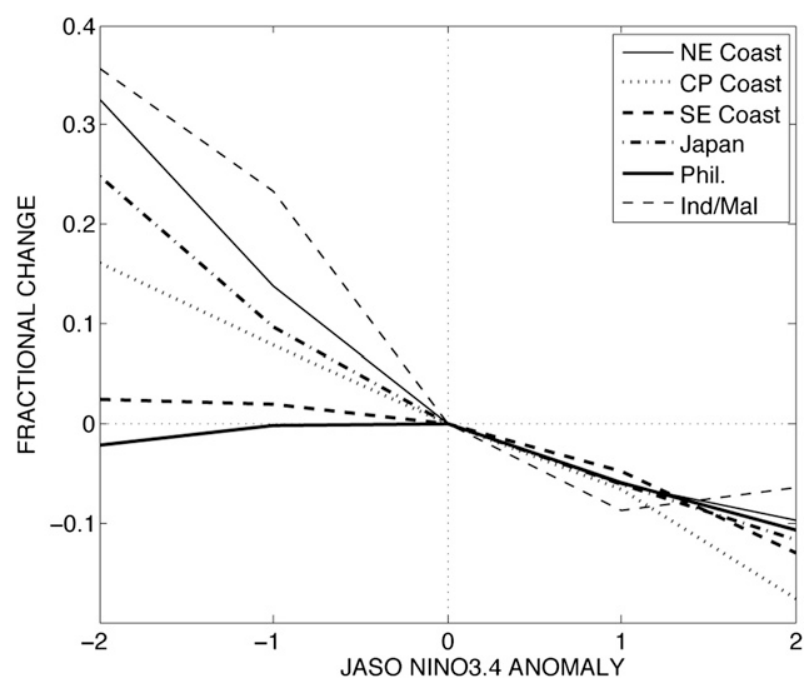

FIG. 15. Fractional change in landfall for political regions given a stationary value of ENSO anomaly.
This idea will be explored further once an intensity model is implemented.

Acknowledgments. This work was supported in part by a grant from the NASA Applied Sciences program. The authors are grateful to Suzana Camargo and Anthony Del Genio for helpful comments on this work.

\section{REFERENCES}

Barnston, A. G., M. Chelliah, and S. B. Goldenberg, 1997: Documentation of a highly ENSO-related SST region in the equatorial Pacific. Atmos.-Ocean, 35, 367-383.

Camargo, S. J., and A. H. Sobel, 2005: Western North Pacific tropical cyclone intensity and ENSO. J. Climate, 18, 29963006.

— K. A. Emanuel, and A. H. Sobel, 2007a: Use of a genesis potential index to diagnose ENSO effects on tropical cyclone genesis. J. Climate, 20, 4819-4834.

- A. W. Robertson, S. J. Gaffney, P. Smyth, and M. Ghil, 2007b: Cluster analysis of typhoon tracks. Part II: Large-scale circulation and ENSO. J. Climate, 20, 3654-3676.

Chan, J. C. L., 1985: Tropical cyclone activity in the northwest Pacific in relation to the El Niño/Southern Oscillation phenomenon. Mon. Wea. Rev., 113, 599-606.

- 2000: Tropical cyclone activity over the western North Pacific associated with El Niño and La Niña events. J. Climate, 13, 2960-2972.

_ 2005: Interannual and interdecadal variations of tropical cyclone activity over the western North Pacific. Meteor. Atmos. Phys., 89, 143-152.

_ 2006: Comment on "Changes in tropical cyclone number, duration, and intensity in a warming environment." Science, 311, 1713.

_ , and J. Shi, 2000: Frequency of typhoon landfall over Guangdong Province of China during the period 1470-1931. Int. J. Climatol., 20, 183-190.

— Pacific typhoon activity from an observational perspective. J. Climate, 17, 4590-4602.

Chia, H.-H., and C. F. Ropelewski, 2002: The interannual variability in the genesis location of tropical cyclones and the northwest Pacific. J. Climate, 15, 2934-2944.

Chu, P., and J. Wang, 1998: Modeling return periods of tropical cyclone intensities in the vicinity of Hawaii. J. Appl. Meteor., 39, 951-960.

Darling, R., 1991: Estimating probabilities of hurricane wind speeds using a large-scale empirical model. J. Climate, 4, 1035-1046. 
Dong, K., 1988: El Niño and tropical cyclone frequency in the Australian region and the northwest Pacific. Aust. Meteor. Mag., 36, 219-255.

Drayton, M., 2000: A stochastic "basin-wide" model of Atlantic hurricanes. Proc. 24th Conf. on Hurricanes and Tropical Meteorology, Ft. Lauderdale, FL, Amer. Meteor. Soc., 17A.3.

Elsner, J. B., and K. B. Liu, 2003: Examining the ENSO-typhoon hypothesis. Climate Res., 25, 43-54.

—, R. J. Murnane, and T. H. Jagger, 2006: Forecasting U.S. hurricanes 6 months in advance. Geophys. Res. Lett., 33, L10704, doi:10.1029/2006GL025693.

Emanuel, K., S. Ravela, E. Vivant, and C. Risi, 2006: A statistical deterministic approach of hurricane risk assessment. Bull. Amer. Meteor. Soc., 87, 299-314.

Fudeyasu, H., S. Iizuka, and T. Matsuura, 2006: Impact of ENSO on landfall characteristics of tropical cyclones over the western North Pacific during the summer monsoon season. Geophys. Res. Lett., 33, L21815, doi:10.1029/2006GL027449.

Hall, T. M., and S. Jewson, 2007: Statistical modeling of North Atlantic tropical cyclone tracks. Tellus, 59A, 486-498.

Harr, P. A., and R. L. Elsberry, 1991: Tropical cyclone track characteristics as a function of large-scale circulation anomalies. Mon. Wea. Rev., 119, 1448-1468.

James, M. K., and L. B. Mason, 2005: Synthetic tropical cyclone database. J. Waterw. Port Ocean Eng., 131 (4), 181-192.
Knapp, K. R., M. C. Kruk, D. H. Levinson, H. J. Diamond, and C. J. Neumann, 2010: The International Best Track Archive for Climate Stewardship (IBTrACS): Unifying tropical cyclone best track data. Bull. Amer. Meteor. Soc., 91, 363-376.

Rumpf, J., H. Weindl, P. Höppe, E. Rauch, and V. Schmidt, 2007: Stochastic modeling of tropical cyclone tracks. Math. Methods Oper. Res., 66, 475-490.

Sabbatelli, T. A., and M. E. Mann, 2007: The influence of climate state variables on Atlantic tropical cyclone occurrence rates. J. Geophys. Res., 112, D17114, doi:10.1029/2007JD008385.

Saunders, M. A., R. E. Chandler, C. J. Merchant, and F. P. Roberts, 2000: Atlantic hurricanes and NW Pacific typhoons: ENSO spatial impacts on occurrence and landfall. Geophys. Res. Lett., 27, 1147-1150.

Vickery, P. J., P. F. Skerlj, and L. A. Twisdale, 2000: Simulation of hurricane risk in the U.S. using an empirical track model. J. Struct. Eng., 126, 1222-1237.

Wang, B., and J. C. L. Chan, 2002: How strong ENSO events affect tropical storm activity over the western North Pacific. J. Climate, 15, 1643-1658.

Wu, M. C., W. L. Chang, and W. M. Leung, 2004: Impacts of El Niño-Southern Oscillation events on tropical cyclone landfalling activity in the western North Pacific. J. Climate, 17, $1419-1428$ 